\title{
A designed equine herpes thymidine kinase (EHV4 TK) variant improves ganciclovir-induced cell-killing
}

\author{
Theresa McSorley ${ }^{\mathrm{a}, \mathrm{b}, *}$, Stephan Ort ${ }^{\mathrm{a}, 1}$, Christian Monnerjahn ${ }^{\mathrm{a}, 2}$, Manfred Konrad ${ }^{\mathrm{a}, *}$ \\ ${ }^{a}$ Max-Planck-Institute for Biophysical Chemistry, Research Group Enzyme Biochemistry, 37077 Göttingen, Germany \\ ${ }^{\mathrm{b}}$ Institute for Geophysics, Georg-August University, 37077 Göttingen, Germany
}

\section{A R T I C L E I N F O}

\section{Article history:}

Received 5 September 2013

Accepted 19 November 2013

Available online 4 December 2013

\section{Keywords:}

Herpes simplex virus thymidine kinase

Equine herpes virus-4 thymidine kinase

Ganciclovir

Suicide gene therapy

Enzyme prodrug activation

\begin{abstract}
A B S T R A C T
The limitations of the ganciclovir (GCV)/herpes simplex virus thymidine kinase (HSV1 TK: EC 2.7.1.21) system as a suicide gene therapy approach have been extensively studied over the years. In our study, we focused on improving the cytotoxic profile of the GCV/equine herpes virus- 4 thymidine kinase (EHV4 TK: EC 2.7.1.21) system. Our approach involved the structure-guided mutagenesis of EHV4 TK in order to switch its ability to preferentially phosphorylate the natural substrate deoxythymidine (dT) to that of GCV. We performed steady-state kinetic analysis, genetic complementation in a thymidine kinasedeficient Escherichia coli strain, isothermal titration calorimetry, and analysis of GCV-induced cell killing through generation of HEK 293 stable cell-lines expressing EHV4 TK mutants and wild-type EHV4 TK. We found that the EHV4 TK S144H-GFP mutant preferentially phosphorylates GCV and confers increased GCV-induced cytotoxicity compared to wild-type EHV4 TK.
\end{abstract}

(c) 2013 Elsevier Inc. All rights reserved.

\section{Introduction}

The transduction of tumor cells with the herpes simplex virus thymidine kinase (HSV1 TK: EC 2.7.1.21) combined with the prodrug ganciclovir (GCV) is a well characterized form of suicide gene therapy first introduced over 25 years ago [1]. This therapy was conceived on the basis that the guanosine analog GCV is not, or only poorly, accepted as substrate by the cellular thymidine kinases, or other nucleoside kinases, that would catalyze the first phosphorylation step in metabolic activation of this prodrug. Therefore GCV would be physiologically inert, unless converted to the tri-phosphorylated form that acts as substrate of DNA polymerases. The expression of HSV1 TK in tumor cells allows site-specific activation of GCV. Activated GCV thus only causes cell death at the tumor site by DNA damage, and has very little effect on the surrounding cells/tissue. So far, many strategies have targeted the treatment of aggressive malignant brain tumors such as

\footnotetext{
Abbreviations: ACV, acyclovir; dC, deoxycytidine; dG, deoxyguanosine; dU, deoxyuridine; EHV4, equine herpes virus type 4; GCV, ganciclovir; HSV1, herpes simplex virus type 1 ; IPTG, isopropyl $\beta$-D-1-thiogalactopyranoside; PMSF, phenylmethylsulfonyl fluoride; dT, thymidine; TK, thymidine kinase.

* Corresponding authors.

E-mail addresses: tmcsorl@gwdg.de, theresamcsorley@hotmail.com (T. McSorley), mkonrad@gwdg.de (M. Konrad).

${ }^{1}$ Present address: SynteractHCR Deutschland GmbH, 80636 München, Germany.

2 Present address: Salutas Pharma GmbH, 39179 Barleben, Germany.
}

glioblastoma multiforme, however, overall they have had only moderate success in clinical trials [1-5]. Most recently, the HSV1 TK/GCV-based enzyme/prodrug system has been put forward as an efficient strategy for stem cell-based gene therapy [6,7].

Different factors are thought to contribute to the limitations of the HSV1 TK/GCV strategy, one being that GCV is not efficiently phosphorylated by HSV1 TK. Following the delivery of HSV1 TK cDNA to tumor cells, GCV is phosphorylated to $5^{\prime}$-monophosphate (GCV-MP) by HSV1 TK, and subsequently converted to 5'diphosphate (GCV-DP) and 5'-triphosphate (GCV-TP) by cellular kinases [8]. The GCV-TP metabolite has been implicated in the initiation of the apoptosis pathway, though the exact mechanism whereby the cell becomes apoptotic is still unknown. Briefly, GCV$\mathrm{TP}$ is incorporated in the elongating DNA chain resulting in S-phase cell-cycle arrest, and/or GCV-TP competitively inhibits the cellular DNA polymerases, resulting in inhibition of DNA synthesis, and ultimately apoptosis [9-15].

Over the years, extensive kinetic analysis has been performed on HSV1 TK with the aim to improve its kinetic properties with nucleoside analogs as substrates, and thus improve its tumor cell killing capacity. As well as GCV, HSV1 TK also recognizes the nucleoside analogs acyclovir (ACV), penciclovir (PCV), and brivudine (BVDU) as substrates, and accepts the natural nucleosides deoxycytidine $(\mathrm{dC})$, deoxyguanosine $(\mathrm{dG})$, deoxyuridine (dU), deoxythymidine (dT) and thymidylate (dTMP) as substrates $[16,17]$. Thus, in the tumor cell context, this promiscuity may also influence the GCV cell-killing efficiency due to competition 
between GCV and the natural substrates of HSV1 TK. As HSV1 TK has a highest affinity (lowest $K_{\mathrm{m}}$ ) for $\mathrm{dT}$, it has been proposed that enabling HSV1 TK to preferentially bind and phosphorylate GCV rather than dT, would improve the cytotoxic effect of the HSV1 TK/ GCV enzyme/prodrug combination [18-22]. On this basis, random mutagenesis and rational protein engineering studies have focused on generating HSV1 TK variants, which would selectively and efficiently phosphorylate GCV [18-22]. Several HSV1 TK mutants have been shown to have compromised dT activity while still possessing the ability to phosphorylate GCV.

For our study, we focused on structure-guided mutagenesis of equine herpes thymidine kinase (EHV4 TK: EC 2.7.1.21). Previously, Loubière et al. compared thymidine kinases expressed by other members of the herpes virus family and found that both murine and human cell-lines transfected with EHV4 TK, which is expressed by the equine herpes virus type-4, resulted in 3-12 times more GCV-induced cytotoxicity compared to HSV1 TKtransfected cells [23]. Based on the EHV4 TK crystal structure and sequence homologies to HSV1 TK, we have generated EHV4 TK mutants also with the aim to either ablate or reduce the affinity of $\mathrm{dT}$ binding and ultimately increase the GCV-induced cell killing sensitivity [24]. We found that the EHV4 TK S144H mutant resulted in residual dT activity and following expression in HEK 293 cells imposed increased GCV sensitivity compared to wildtype EHV4 TK.

\section{Materials and methods}

\subsection{Materials}

Oligonucleotides were purchased from IBA GmbH (Göttingen, Germany). PCR reagents were from PeqLab (Erlangen, Germany) and restriction enzymes from (New England Biolabs, Ipswich, MA). Dulbecco's Modified Eagle Medium (DMEM) was purchased from Sigma-Aldrich (Steinheim, Germany), L-glutamine and fetal calf serum (FCS) were purchased from PAA Laboratories GmbH (Cölbe, Germany). G-418 solution, FuGene ${ }^{\circledR}$ HD Transfection Reagent and Cell Proliferation Reagent WST-1 were purchased from Roche (Mannheim, Germany). Nucleosides, ACV and GCV were purchased from Sigma-Aldrich.

Anti-GFP IgG antibody was a gift from Dr. Dieter Schmitt (MPI for Biophysical Chemistry, Göttingen). Anti- $\alpha$-tubulin mAb was a gift from Prof. Mary Osborn (MPI for Biophysical Chemistry, Göttingen). Anti-nuclear poly(ADP-ribose) polymerase (PARP) IgG was purchased from Cell Signaling (Danvers, MA), IgG antibodies conjugated to horseradish-peroxidase were purchased from DiaNova (Hamburg, Germany).

\subsection{Molecular biology}

The plasmids pUT599[HSV1 TK] and pUT699[EHV4 TK] have been described previously [23]. A maltose-binding protein (MBP)-polyhistidine double affinity fusion of EHV4 TK was obtained by cloning into a modified pMAL c2 vector (New England Biolabs, Ipswich, MA) a fragment of two annealed oligos ( $5^{\prime}$-cgc acc atc acc atc acc acc agg tcg gtg gtg gcg gtg gct tgg ttc cgc gtg get ctc ata tgg aat tcg-3', and $5^{\prime}$-gat ccg aat tcc ata tga gag cca cgc gga acc aag cca ccg cca cca ccg acc tgg tgg tga tgg tga tgg tgc gag $c\left(-3^{\prime}\right)$ to generate a linker coding for the amino acid sequence HHHHHHQVGGGGGLVPRGS with an N-terminal SacI site and C-terminal NdeI, EcoRI and BamHI sites. The fragment was ligated into pMAL c2 via SacI and BamHI, generating the pMAL K4 vector. This MBP-His $_{6}$-tag vector allowed purification of soluble protein by metal affinity chromatography and efficient thrombin protease cleavage (LVPR'GS site) of the fusion protein.
Site-specific mutations of EHV4 TK were generated by overlapextension PCR using overlapping oligonucleotide sets: (EHV4 TK $5^{\prime}$-gga att cca tat ggc tgc ttg cgt ac-3', and EHV4 TK $3^{\prime}$-gga tcc tca gac gec cat ctc cgc-5'; EHV4 TK A143Y $5^{\prime}$-ccg gtc tact ct acc gta tgc ttt cca g- $3^{\prime}$, and $3^{\prime}$-tac ggt aga gta gac cgg gtg gcg-5'; EHV4 TK S144H $5^{\prime}$-gtc gec cat acc gta tgc ttt cca gc-3', and EHV4 TK S144H 3'-tac ggt atg ggc gac cgg gtg-3') [25]. The EHV4 TK $5^{\prime}$ oligos contained a BamHI site, and the EHV4 TK $3^{\prime}$ oligos contained an NdeI site for cloning into the modified pMAL $\mathrm{c} 2$ vector. HSV1 TK was cloned into a pET14b vector via NdeI and BamHI sites to create an N-terminal $\mathrm{His}_{6}$-tag fusion protein. For expression in mammalian cells, EHV4 TK wild-type and the EHV4 TK mutants were sub-cloned from the pMAL c2 vector by PCR to obtain fragments with $\mathrm{N}$-terminal NdeI and C-terminal BamHI sites. Fragments were then ligated into pEGFP-C1 and pEGFP-N1 vectors (Clontech, Saint-Germain-enLaye, France) modified to contain NdeI in the multiple cloning site.

\subsection{Protein expression and purification}

Escherichia coli BL21 C41 (DE3) was used for recombinant protein production. Cells were grown in $L B$ medium to an $\mathrm{OD}_{600}$ of $0.7-0.9$. Protein overexpression was induced with $0.5-1 \mathrm{mM}$ IPTG. Cells were incubated at $25^{\circ} \mathrm{C}$ for $7 \mathrm{~h}$ (HSV1 TK) or at $18{ }^{\circ} \mathrm{C}$ for $24 \mathrm{~h}$ (EHV4 TK), and then harvested by centrifugation. Cell pellets were suspended in buffer A ( $50 \mathrm{mM}$ Hepes, pH 7.2, $500 \mathrm{mM} \mathrm{NaCl}, 10 \%$ (v/ v) glycerol, $1 \%(\mathrm{v} / \mathrm{v})$ Triton X-100, $5 \mathrm{mM} ß$-mercaptoethanol, $0.5 \mathrm{mM}$ PMSF), and lysed by sonication. After centrifugation of the homogenate $\left(12,000 \times \mathrm{g}, 30 \mathrm{~min}, 4^{\circ} \mathrm{C}\right)$, the supernatant was incubated overnight with Ni-NTA agarose (Qiagen, Hilden, Germany). The resin with bound $\mathrm{His}_{6}$-tagged protein was washed with buffer A containing $10 \mathrm{mM}$ imidazole. The enzyme moieties were released by thrombin-cleavage $(0.5$ units $/ \mathrm{ml})$ of matrixbound fusion protein, size-checked by PAGE, and quantified using the Bio-Rad dye-binding assay.

\subsection{Enzyme activity measurements}

An NADH-dependent enzyme coupled assay [26] performed on a Kontron Uvikon 943 double beam UV/VIS spectrophotometer was used to determine the thymidine kinase activities by recording the decrease of NADH absorption measured at $340 \mathrm{~nm}$, at $25^{\circ} \mathrm{C}$ in the assay buffer (100 mM Tris, pH 7.5, $100 \mathrm{mM} \mathrm{KCL,} 10 \mathrm{mM} \mathrm{MgCl}_{2}$, and $1 \mathrm{mM} \mathrm{ATP}$ ). For $k_{\mathrm{cat}}$ and $K_{\mathrm{m}}$ determination, the following range of concentrations were used: GCV, 20-500 $\mu \mathrm{M}$; D-dT, 1-500 $\mu \mathrm{M}$; ACV, 20-500 $\mu \mathrm{M}$.

\subsection{TK-deficiency rescue assay}

The E. coli strain KY895 ( $\lambda^{-}$, tdk-1. IN (rrnD-rrnE)1, ilv-276; $E$. coli genetic stock center nr. 4842) lacks TK activity, but it is able to synthesize thymidine mono-phosphate (TMP) from deoxyuridine monophosphate (dUMP) through the thymidylate synthase (TS) pathway. A block of this pathway by the TS inhibitor 5-fluorodeoxyuridine (5-FdUrd) prevents growth of E. coli KY895. Complementation of the missing TK activity by plasmid-encoded enzymes was tested by transforming this strain with $E$. coli TK, HSV1 TK, EHV4 TK, or EHV4 TK A143Y cloned into pGEX RB and replating transformants onto selection agar (2\% peptone, $0.5 \%$ $\mathrm{NaCl}, 0.2 \%$ glucose, $2 \mu \mathrm{g} / \mathrm{ml}$ Thd, $12.5 \mu \mathrm{g} / \mathrm{ml}$ Urd, $100 \mu \mathrm{g} / \mathrm{ml}$ ampicillin, $10 \mu \mathrm{g} / \mathrm{ml}$ 5-FdUrd).

\subsection{Isothermal titration calorimetry}

Isothermal titration calorimetry (ITC) measurements were performed in an OMEGA titration calorimeter (Microcal). The reference cell was filled with water. All solutions were degassed 
prior to experiments. Protein samples were dialyzed over night against buffer $A$, concentrated to $20-25 \mu \mathrm{M}$ using Vivaspin concentrators (Sartorius, Göttingen, Germany), and loaded into the calorimeter cell ( $1.45 \mathrm{ml}$ volume). Thymidine was dissolved in the dialysis buffer at a concentration 25-fold higher than that of EHV4 TK or EHV4 TK A143Y. The thymidine solution was titrated in $8 \mu \mathrm{l}$ aliquots (12 injections, $3 \mathrm{~min}$ intervals between injections) EHV4 TK or EHV4 TK A143Y. Raw data were integrated with the Origin ${ }^{\circledR}$ software supplied with the instrument.

\subsection{Cell-culture and stable cell-line generation}

The HEK 293 cell-line was cultured in standard complete DMEM medium, which is DMEM supplemented with $10 \%$ heatinactivated FCS and L-glutamine and maintained at $37{ }^{\circ} \mathrm{C}$ in humidified atmosphere containing $5 \% \mathrm{CO}_{2}$.

To generate HEK 293 stable cell-lines, cells were seeded into $10 \mathrm{~cm}$ dishes and transfected using FuGene ${ }^{\circledR}$ HD Transfection Reagent according to the manufacturer's instructions. The standard complete DMEM medium was exchanged to complete DMEM supplemented with $900 \mu \mathrm{g} / \mathrm{ml} \mathrm{G}-418$. After 7-10 days, colonies were observed, isolated using a pipette tip under an inverted light-microscope, and transferred to 12-well plates. After 2-3 days, the stable colonies were monitored using a fluorescencemicroscope and further sub-cultured. The established stable celllines were maintained in complete DMEM medium containing $300 \mu \mathrm{g} / \mathrm{ml} \mathrm{G}-418$.

\subsection{Cell proliferation/survival}

For GCV dose-response analysis, HEK-stable cell-lines were seeded in 96 well plates. GCV in the range of $1 \mathrm{nM}-100 \mu \mathrm{M}$ was added, with triplicate wells for each concentration. After $96 \mathrm{~h}$, WST-1 proliferation assays were performed according to the manufacturer's instructions and analyzed on a $\mu$ Quant microplate spectrophotometer (Bio-Tek Instruments) at $450 \mathrm{~nm}$.

The assay results at different concentrations of GCV were compared to the wells without GCV (control) and the percentage cell survival plotted. The GCV EC 50 values were calculated with GraphPad Prism 5 software (Nonlinear regression fitting; standard slope (Hill-slope $=1.0)$ ).

\subsection{Western-blot analysis}

The stable HEK293 cell-lines cultured in 6-well plates were lysed with standard lysis-buffer $\left(10 \mathrm{mM} \mathrm{K}_{2} \mathrm{HPO}_{4}, 150 \mathrm{mM} \mathrm{NaCl}\right.$ (pH 7.4), 5 mM EDTA, 5 mM EGTA, 0.5 mM PMSF, and protease inhibitor cocktail). Cell lysates were snap-frozen in liquid nitrogen, thawed, subjected to 10 strokes through a 20-gauge needle and centrifuged for $10 \mathrm{~min}$ at $13,000 \mathrm{rpm}, 4^{\circ} \mathrm{C}$. The total protein concentration of supernatants was measured by Bradford assay. Samples of the cell-lysate supernatants were prepared in $6 \times$ Laemmli buffer, heated for $5 \mathrm{~min}$ at $95^{\circ} \mathrm{C}$. The supernatant samples were subjected to SDS-PAGE in $12.5 \%$ acrylamide gels (15-20 $\mu \mathrm{g}$ total protein per lane), and transferred to nitrocellulose.

Nitrocellulose membranes were incubated with Ponceau S stain to assess transfer and loading, and then blocked with 5\% skimmed milk for $1 \mathrm{~h}$ at room temperature. The membranes were incubated with anti-GFP IgG antibody in $1 \%$ milk for $2-3 \mathrm{~h}$ at room temperature, washed and incubated with horseradish peroxidase-conjugated anti-rabbit IgG in $1 \%$ milk for $1 \mathrm{~h}$ at room temperature. The membranes were re-probed with anti- $\alpha$-tubulin $\mathrm{mAb}$ to show loading of lanes. Quantitative analysis of Westernblot data was performed using Image J 1.44p software (http:// rsbweb.nih.gov/ij/).
For the analysis of poly(ADP-ribose) polymerase (PARP) cleavage, the supernatant samples were subjected to SDS-PAGE in $10 \%$ acrylamide gels, blotted, membranes stained with Ponceau $\mathrm{S}$ incubated with anti-PARP IgG overnight at $4{ }^{\circ} \mathrm{C}$. Anti-PARP IgG detects both full length PARP (116 kDa) and the large caspasecleaved fragment of PARP ( $89 \mathrm{kDa})$.

\subsubsection{Confocal microscopy}

Cells were grown on $20 \mathrm{~mm}$ glass cover-slips, fixed with $4 \%$ paraformaldehyde for $10 \mathrm{~min}$ at room temperature, incubated with DAPI ( $300 \mathrm{nM}$ ) for $2 \mathrm{~min}$ at room temperature, and the coverslips were mounted in Mowiol. The images were taken with $25 \mathrm{x}$ objective on a Zeiss LSM5 confocal microscope.

\section{Results}

\subsection{Kinetic analysis comparison of wild-type HSV1 TK, wild-type EHV4 TK, and EHV4 TK mutants}

In the case of HSV1 TK, Balzarini et al. have generated HSV1 TK mutants with the aim of ablating dT kinase activity, yet preserving purine nucleoside analog activity [22]. Through a structural modeling approach, they found individual amino acid mutations, where alanine residues substituted to phenylalanine or histidine at positions 167 or 168 (A167F or A168H, respectively) successfully compromised dT activity, but preserved GCV activity. As the tertiary structures of HSV1 TK and EHV4 TK overlap, and they share many similarities in secondary structure, we proposed that mutations at the corresponding sites in EHV4 TK would have similar effects [24]. As shown in the amino acid alignment of HSV1 TK and EHV4 TK, positions 167 and 168 in HSV1 TK correspond with positions 143 and 144 in EHV4 TK (Fig. 1A). We constructed two different mutations, A143Y and S144H, also with the aim to hinder dT activity, yet preserve GCV activity. As the crystal structure of EHV4 TK bound to its natural substrate thymidine (dT) has been solved [24], we used this structural information to create diagrams, which reveal the structural positions of both the A143Y and $\mathrm{S} 144 \mathrm{H}$ mutations (Fig. 1B and C). In order to visualize the dT binding site more clearly, alpha-helices 1 and $12(\alpha 1$ and $\alpha 12)$ and beta-sheet 5 ( $\beta 5)$ were removed. In the case of the A143Y mutation, the steric clash of the larger aromatic side chain of tyrosine with the dT ring can clearly be seen (Fig. 1B). The S144H mutation is presumed to affect dT binding through a steric hindrance created by the histidine ring (Fig. 1C).

The steady-state kinetics of each mutant, EHV4 TK A143Y and EH4TK S144H was analyzed and compared to wild-type EHV4 TK and HSV1 TK (Table 1 ). The $K_{\mathrm{m}}$ values and turn-over rates were measured for dT and GCV. As previously reported, dT is the preferred substrate for both HSV1 TK WT and EHV4 TK WT ( $K_{\mathrm{m}}=12 \mu \mathrm{M}$, and $5.8 \mu \mathrm{M}$, respectively). Of the two EHV4 TK mutants, the EHV4 TK S144H mutation resulted in a much higher $K_{\mathrm{m}}(>500 \mu \mathrm{M})$, and the EHV4 TK A143Y mutation resulted in the total loss of dT activity. To observe whether a low residual dT activity for EHV4 TK A143Y existed which was not detected by the steady-state kinetic measurements, a complementation assay for TK activity in E. coli was performed, and indeed revealed that EHV4 TK A143Y lost the capacity to genetically complement the thymidine kinase-deficient mutant strain (Fig. 2(A) and (B)). In this assay, 5-FdUrd is included in the selection media, and under these conditions, the generation of TMP becomes dependent on an active thymidine kinase enzyme. Here, E. coli TK, HSV1 TK, and EHV4 TK were able to rescue the TK-deficiency of the E. coli KY895 strain; however, EHV4 TK A143Y did not (Fig. 2(A) and (B)). This indicates that the mutant EHV4 TK A143Y enzyme has lost the capacity to phosphorylate the natural substrate thymidine. 
(A)

HSV1TK

EHV4TK

HSV1TK

EHV4TK

HSV1TK

EHV4TK

HSV1TK

EHV4TK

HSV1TK

EHV4TK

HSV1TK

EHV4TK

HSV1TK

EHV4TK
MASYPCHQHASAFDQAARSRGHSNRRTALRPRRQQEATEVRLEQKMPTLLRVY IDGPHGM 60

MA--------------ACVPPGEAPRSASGTPTRRQ------------VTIVRIYLDGVYGI 36

GKTTTTQLLVALGSRDD-IVYVPEPMTYWQVLGASET IANIYTTQHRLDQGEISAGDAAV 119

GKSTTGRVMASAASGGSPTLYFPEPMAYWRTLFETDVISGIYDTQNRKQQGNLAVDDAAL 96

VMT SAQ ITMGMPYAVTDAVLAPHIGGEAGSSHAPPPALTLIFDRHPIAALLCYPAARYLM 179

ITAHYQSRFTTPYLILHDHTCTLFGGNS-LQRGTQPDLTLVFDRHPVASTVCFPAARYLL 155

**

GSMTPQAVLAFVALIPPTLPGTNIVLGALPEDRHIDRLAKRQRPGERLDLAMLAAIRRVY 239

GDMSMCALMAMVATLPREPQGGN IVVTTLNVEEHIRRTRTRARIGEQIDITLIATLRNVY 215

GLLANTVRYLQGGGSWREDWGQLSGTAVPPQGAEPQSNAGPR---PHIGDTLFTLFRAPE 296

FMLVNTCHFLRSGRVWRDGWGELPT SCGAYKHRATQMDAFQERVSPELGDTLFALFKTQE 275

LLAPNGDLYNVFAWALDVLAKRLRPMHVF ILDYDQSPAGCRDALLQLT SGMVQTHVTTPG 356

LLDDRGVILEVHAWALDALMLKLRNLNVF SADLSGTPRQCAAVVESLLP-LMSSTLSDFD 334
(B)
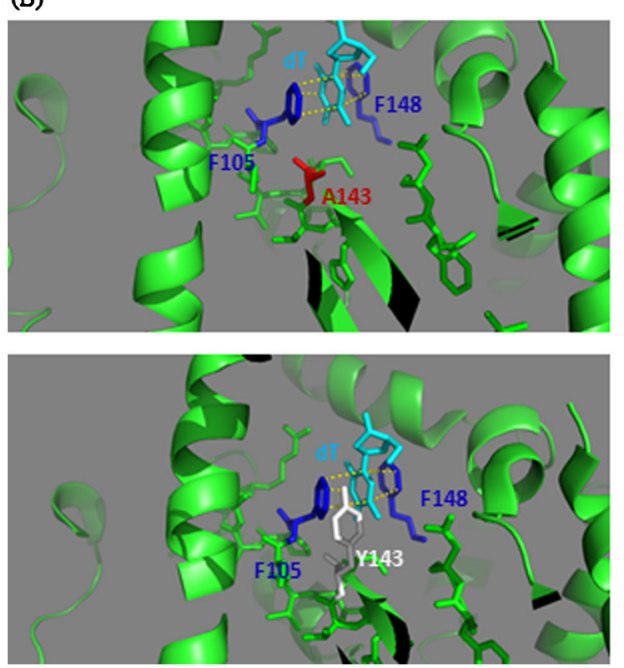

(C)
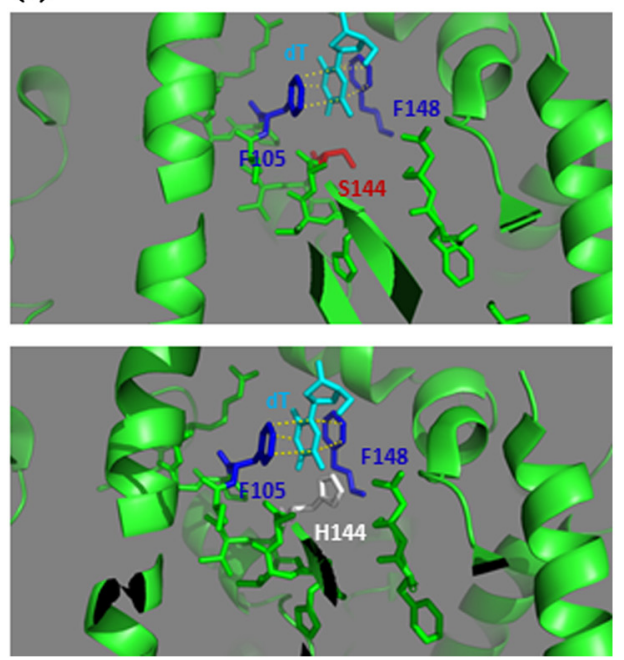

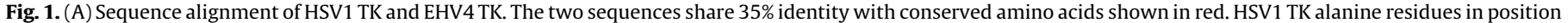

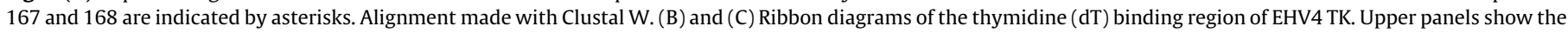

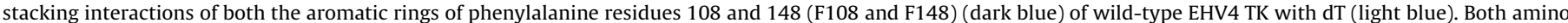

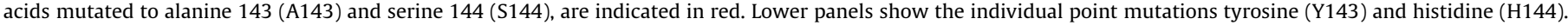

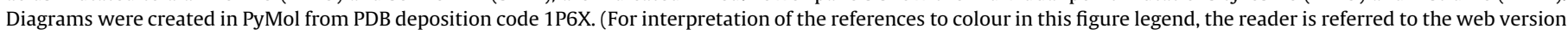
of this article.)

Furthermore, we performed isothermal titration calorimetry (ITC) to analyze if the EHV4 TK A143Y mutant enzyme had lost the ability to bind dT (Fig. 2(C) and (D)). The binding of dT to EHV4 TK WT was found to be exothermic, the downward peaks indicating heat liberation on injection of the ligand solution into the protein solution. In the case of EHV4 TK A143Y, a trace was observed that resulted solely from the ligand dilution, indicating that the mutant protein could no longer interact with $\mathrm{dT}$.

Although EHV4 TK S144H did not lose dT phosphorylation activity, the $K_{\mathrm{m}}$ for dT increased over 100 -fold $\left(K_{\mathrm{m}}>500 \mu \mathrm{M}\right)$. In the case of GCV, compared to EHV4 TK WT $\left(K_{\mathrm{m}}=48.2 \pm 22.3 \mu \mathrm{M}\right)$, the $K_{\mathrm{m}}$ values for both EHV4 TK A143Y and EHV4 TK S144H were increased 5-fold and 10-fold $\left(K_{\mathrm{m}}=258 \pm 52 \mu \mathrm{M}\right.$ and $\left.500 \pm 55 \mu \mathrm{M}\right)$. Comparing the turnover rates of EHV4 TK WT, EHV4 TK A143Y, and EHV4 TK S144H $\quad\left(k_{\text {cat }}=0.44 \pm 0.11 \mathrm{~s}^{-1}, \quad 0.42 \pm 0.063 \mathrm{~s}^{-1}\right.$, and $1.37 \pm 0.035 \mathrm{~s}^{-1}$, respectively) with GCV as substrate, and calculating the enzyme efficiencies $\left(k_{\text {cat }} / K_{\mathrm{m}}=9.0 \pm 8.3 \times 10^{3} \mathrm{M}^{-1} \mathrm{~s}^{-1}\right.$, $1.6 \pm 0.41 \times 10^{3} \mathrm{M}^{-1} \mathrm{~s}^{-1}$, and $2.7 \pm 0.3 \times 10^{3} \mathrm{M}^{-1} \mathrm{~s}^{-1}$, respectively), both mutants are 3 to 4 -fold less efficient at phosphorylating GCV. 
Table 1

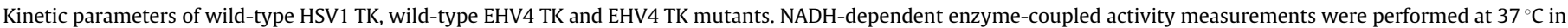

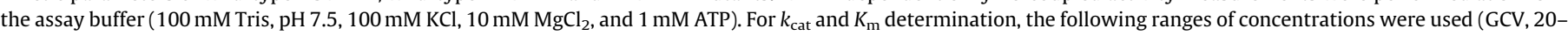

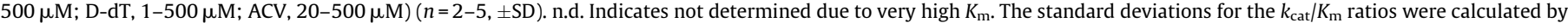
Propagation of Error.

\begin{tabular}{|c|c|c|c|c|c|c|}
\hline & \multicolumn{3}{|l|}{ Thy } & \multicolumn{3}{|l|}{ GCV } \\
\hline & $k_{\text {cat }}\left(\mathrm{s}^{-1}\right)$ & $K_{\mathrm{m}}(\mu \mathrm{M})$ & $k_{\text {cat } /} / K_{\mathrm{m}}\left(\mathrm{M}^{-1} \mathrm{~s}^{-1}\right)$ & $k_{\text {cat }}\left(\mathrm{s}^{-1}\right)$ & $K_{\mathrm{m}}(\mu \mathrm{M})$ & $k_{\text {cat }} / K_{\mathrm{m}}\left(\mathrm{M}^{-1} \mathrm{~s}^{-1}\right)$ \\
\hline HSV1-TK & $0.43 \pm 0.09$ & $12.0 \pm 3.7$ & $35 \times 10^{3} \pm 13.0$ & $0.17 \pm 0.1$ & $36 \pm 10.2$ & $4.7 \times 10^{3} \pm 3.1$ \\
\hline EHV4 TK WT & $0.56 \pm 0.29$ & $5.8 \pm 0.09$ & $97 \times 10^{3} \pm 50$ & $0.44 \pm 0.11$ & $48.2 \pm 22.3$ & $9.0 \times 10^{3} \pm 8.3$ \\
\hline EHV4 TK A143Y & n.d. & n.d. & n.d. & $0.42 \pm 0.063$ & $258 \pm 52.0$ & $1.6 \times 10^{3} \pm 0.41$ \\
\hline EHV4 TK S144H & $0.07 \pm 0.03$ & $>500$ & $<0.14 \times 10^{3}$ & $1.37 \pm 0.035$ & $500 \pm 55.0$ & $2.7 \times 10^{3} \pm 0.3$ \\
\hline
\end{tabular}

\subsection{Generation of stable EHV4 TK, EHV4 TK A143Y, and EHV4 TK} S144H expressing HEK 293 cell-lines

Based on our steady-state kinetic results for the EHV4 TK mutants, we aimed to study whether the cell-killing ability of the EHV4 TK A143Y or EHV4 TK S144H mutants with the prodrug GCV is indeed superior to wild-type EHV4 TK. To do this, we generated several stable cell-lines. In order to observe the intracellular location of EHV4 TK WT and the EHV4 TK mutants, we constructed N-terminal GFP fusions of each. Following the transient transfection of HEK 293 cells with GFP-EHV4 TK WT, unusual polymeric structures were seen (data not shown). We attributed this observation to the fact that EHV4 TK is predicted to form a slipknot during folding which involves the $\mathrm{N}$-terminal part of the enzyme [27], and the addition of N-terminal GFP somehow interfered with this folding. Therefore, we created C-terminal GFP fusions instead. In this case, uniform cytosolic and nuclear

(A) LB-Amp medium

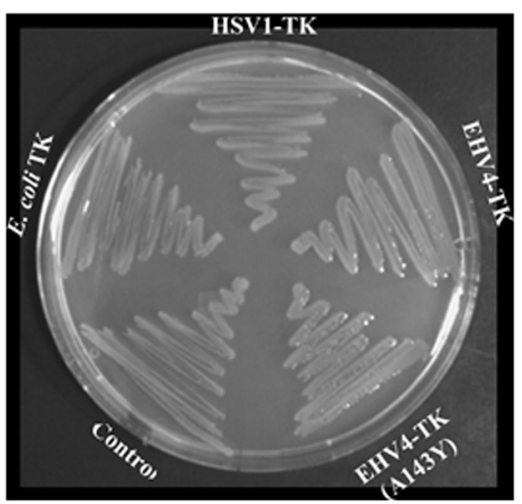

(C) EHV4 TK WT

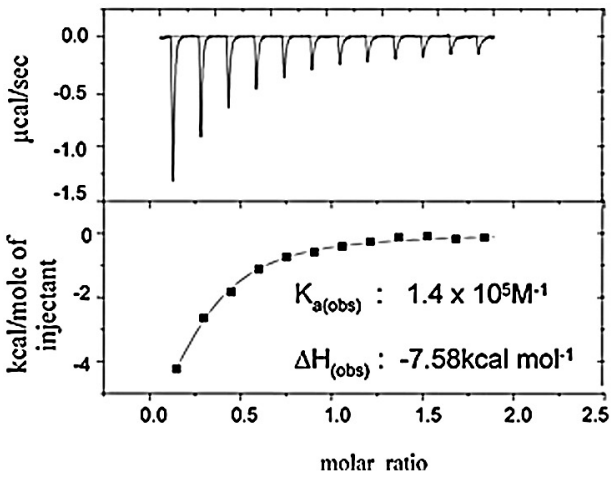

distribution of EHV4 TK WT-GFP, EHV4 TK A143Y-GFP and EHV4 TK S144H-GFP were visualized in HEK 293 cells by confocal microscopy (Fig. 3A), and stable HEK 293 cell-lines expressing each were generated.

The morphology of each stable cell-line and the mother HEK 293 cell-line were similar (data not shown). As both the EHV4 TK A143Y and EHV4 TK S144H mutants have either lost dT activity, or have greatly reduced dT activity compared to EHV4 TK WT, we compared proliferation rates of each stable cell-line and found that they were similar over a period of $48 \mathrm{~h}$ (Fig. 3B).

\subsection{Sensitivity of EHV4 TK WT-GFP, EHV4 TK A143Y-GFP, and EHV4 TK S144H-GFP stable cell-lines to GCV}

Western blot analysis of whole-cell lysates from each stable cell-line revealed the EHV4 TK protein expression levels were not equal (Fig. 4A). The relative EHV4 TK expression compared to

(B) TK-selection medium

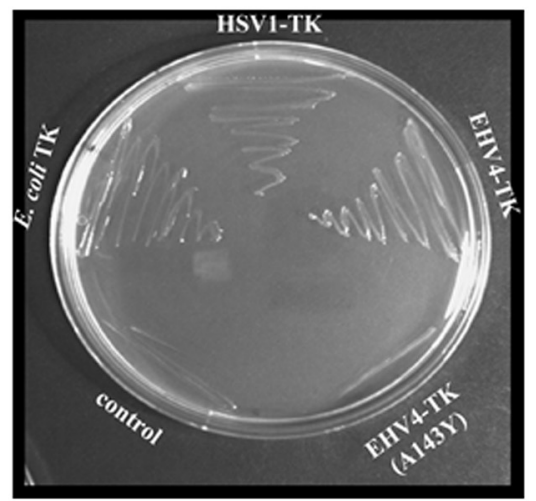

(D) EHV4 TK A143Y

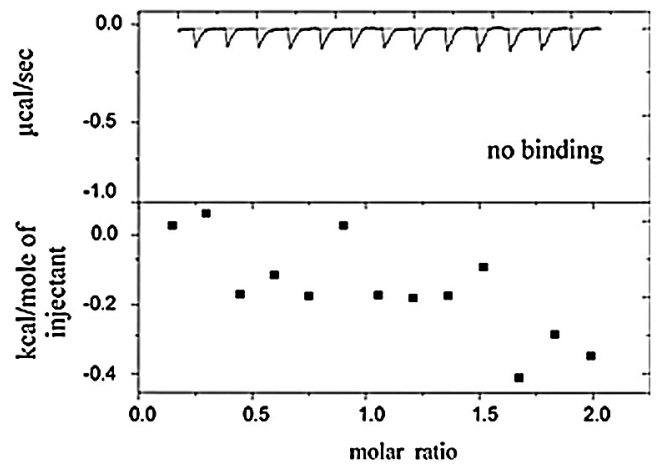

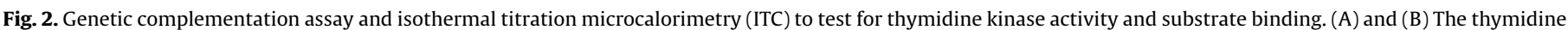

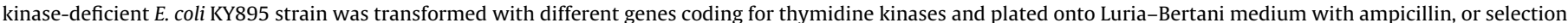

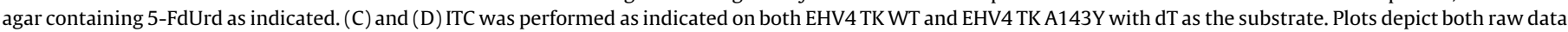

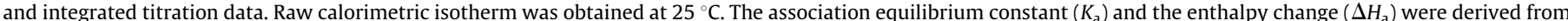

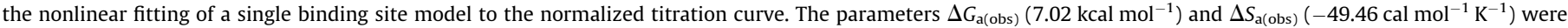
calculated according to $\Delta G_{\mathrm{a}}=-\mathrm{RT} \ln K_{\mathrm{a}}$ and $\Delta S_{\mathrm{a}}=\left(\Delta H_{\mathrm{a}}-\Delta G_{\mathrm{a}}\right) / T$. 
(B)
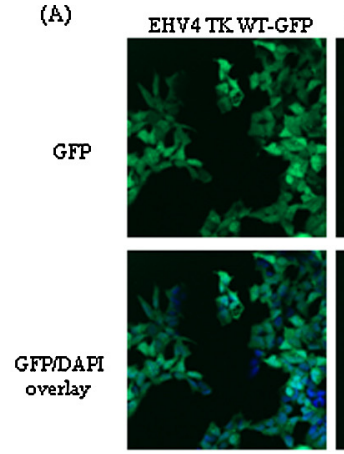

(B)

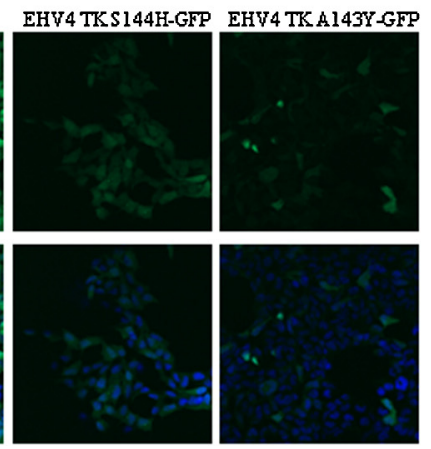

\begin{tabular}{|c|c|c|c|}
\hline & $\begin{array}{c}\operatorname{mean} A_{4502010}-A_{6502 m} \\
O h\end{array}$ & $\begin{array}{c}\operatorname{mean} A_{4502 a n}-A_{6502 a n} \\
24 \mathrm{~h}\end{array}$ & $\begin{array}{c}\operatorname{mean} A_{+50201}-A_{650 \text { man }} \\
48 \mathrm{~h}\end{array}$ \\
\hline EHV4 TKWT-GFP & $0204 \pm 0010$ & $0.443 \pm 0.036$ & $0.724 \pm 0.042$ \\
\hline EHV4 TKA143Y-GFP & $0200 \pm 0.004$ & $0356 \pm 0054$ & $0.761 \pm 0.056$ \\
\hline EHV4 TKS 144H-GFP & $0264 \pm 0.018$ & $0.522 \pm 0.025$ & $0894 \pm 0088$ \\
\hline
\end{tabular}

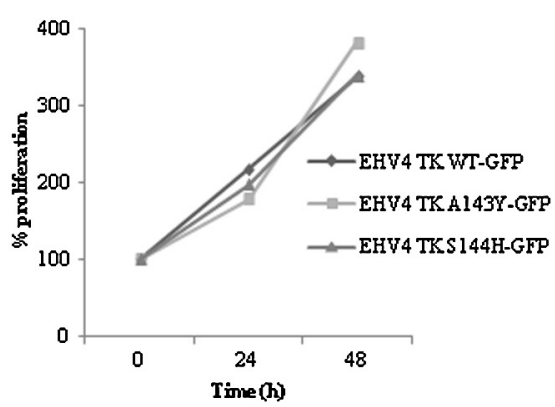

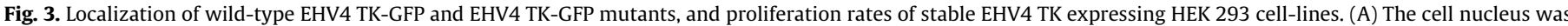

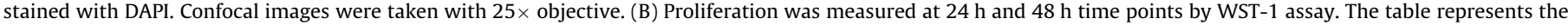
mean absorbance $\left(A_{450 \mathrm{~nm}}-A_{690 \mathrm{~nm}}\right)$ measurements from 4 wells of a 96 -well plate $(n=1, \pm$ SE). Graph represents the percentage proliferation.

$\alpha$-tubulin indicated that the EHV4 TK WT-GFP stable cell-line expressed around 2-fold more EHV4 TK than did the EHV4 TK S144H-GFP stable cell-line, and 6-fold more EHV4 TK than did the EHV4 TK A143Y-GFP stable cell-line.

The sensitivity of the stable cell-lines to GCV was measured by performing dose-response curves (Fig. 4(D) and (F)) and calculating the $\mathrm{EC}_{50}$ values following $96 \mathrm{~h}$ of GCV incubation (Fig. 4B). The $\mathrm{EC}_{50}$ values obtained show that the GCV EC 50 value of EHV4 TK $\mathrm{S} 144 \mathrm{H}(0.1 \pm 0.02 \mu \mathrm{M})$ is lower than that of EHV4 TK WT $(0.6 \pm 0.2 \mu \mathrm{M})$, despite the fact that EHV4 TK WT is more highly expressed. In the case of the EHV4 TK A143Y-GFP stable cell-line, which has the lowest EHV4 TK expression, the $\mathrm{EC}_{50}$ value was measured at $1.8 \pm 0.5 \mu \mathrm{M}$. As an additional control, HEK 293 cells were transiently transfected with pEGFP-N1 vector only (mock) and EHV4 TK WT-GFP, and subjected to $72 \mathrm{~h}$ of GCV-treatment $(1 \mu \mathrm{M}$ and $10 \mu \mathrm{M}$ ) (Fig. 4C). No cytotoxic effect of GCV was observed on the cells expressing GFP only (mock).

We also tested the sensitivity of our stable cell-lines to the nucleoside analog prodrug ACV (Fig. 5). It can be seen that in all cases a high concentration $(500 \mu \mathrm{M})$ is required to achieve over $90 \%$ cell death after $72 \mathrm{~h}$.

\subsection{Time courses of EHV4 TK WT-GFP and EHV4 TK S144H-GFP stable cell-line survival and induction of PARP cleavage following GCV incubation}

Comparing expression levels and $\mathrm{EC}_{50}$ values of EHV4 TK WTGFP, EHV4 TK A143Y-GFP and EHV4 TK S144-GFP stable cell-lines (Fig. 4(A) and (B)), we followed cell-survival and PARP cleavage over a time course for the two most GCV sensitive stable cell-lines. Time-courses of the survival of the EHV4 TK WT-GFP and EHV4 TK S144-GFP stable cell-lines following incubation over a range of GCV concentrations $(0.5 \mu \mathrm{M}, 1 \mu \mathrm{M}, 10 \mu \mathrm{M})$ were performed. At $0.5 \mu \mathrm{M}$ GCV, significant cytotoxicity was only observed with the EHV4 TK S144H-GFP stable cell-line $\left(\mathrm{GCV} \mathrm{EC}_{50}=0.1 \mu \mathrm{M}\right)$, at $24 \mathrm{~h}$ onwards resulting in $25 \%$ cell survival at $96 \mathrm{~h}$ (Fig. 6A). Some cell death also occurred at $0.5 \mu \mathrm{M}$ GCV with the EHV4 TK WT-GFP stable cell-line $\left(\mathrm{GCV} \mathrm{EC} \mathrm{E}_{50}=0.6 \mu \mathrm{M}\right)$, with $70 \%$ cell survival at $96 \mathrm{~h}$. At $1 \mu \mathrm{M} \mathrm{GCV}$, a sharper decline in cell survival was seen with EHV4 TK S144H-GFP as compared to EHV4 TK WT-GFP, with, at 96 h, 15\% and $50 \%$ of cells surviving, respectively (Fig. $6 \mathrm{~B}$ ). Finally, at $10 \mu \mathrm{M}$ GCV concentration, which is far in excess of the GCV EC ${ }_{50}$ s for both EHV4 TK WT-GFP and EHV4 TK S144H-GFP stable cell-lines, the sharpest increase in cell death was observed, and the time-profile of cell death was similar for both cases (Fig. 6C). At 48 h, only 50$60 \%$ of cells survived, and at 96 h only $1-10 \%$ of cells survived.

Time courses were also performed to detect the cleavage of PARP (nuclear poly(ADP-ribose) polymerase), an indicator of down-stream caspase activation and thus apoptosis (Fig. 6D). In both the EHV4 TK WT-GFP and EHV4 TK-S144H stable cell-lines, following $0.5 \mu \mathrm{M} \mathrm{GCV}$ incubation, the most PARP cleavage was observed at the $40 \mathrm{~h}$ time-point.

\section{Discussion}

In the quest to improve the efficacy of the HSV1 TK/GCV-based enzyme/prodrug gene therapy approach, where immunosuppressive concentrations of GCV are needed to give the required response, many different factors have to be considered. One focal point has been to improve the efficiency of the HSV1 TK gene delivery system itself. Previously, adenovirus vectors and retroviral vectors have been used in different stages of clinical trials to treat glioblastomas, without significant success $[3,4,28]$. This lack of success is attributed to the poor transduction of the HSV1 TK gene to the tumor, and the fact that transduction lacks target specificity. Recent approaches, which aim to improve transduction efficiency, to specifically target the tumor, and also to reduce immunogenicity include recombinant non-viral vectors, mesenchymal stem cells, and the use of Bifidobacterium as gene delivery vehicle [29-31]. Furthermore, extensive structural and kinetic 
(A)

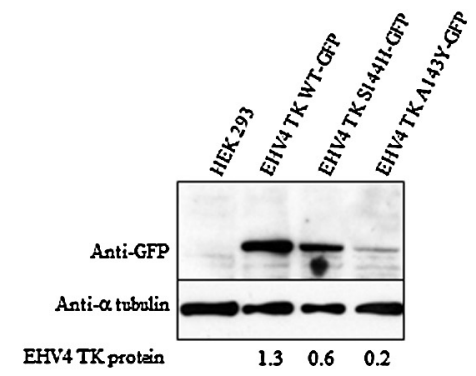

(B)

\begin{tabular}{|c|c|c|}
\hline HFK 293 stable cell-line & GCVEC $_{50}(\mu \mathrm{M})$ & S.E \\
\hline FEK 293 & n.d & \\
\hline EHV4 TK WT-GFP & 0.64 & 0.24 \\
\hline EHV4 TKS144H-GFP & 0.1 & 0.02 \\
\hline EFV4 TKA143Y GFP & 1.83 & 0.48 \\
\hline
\end{tabular}
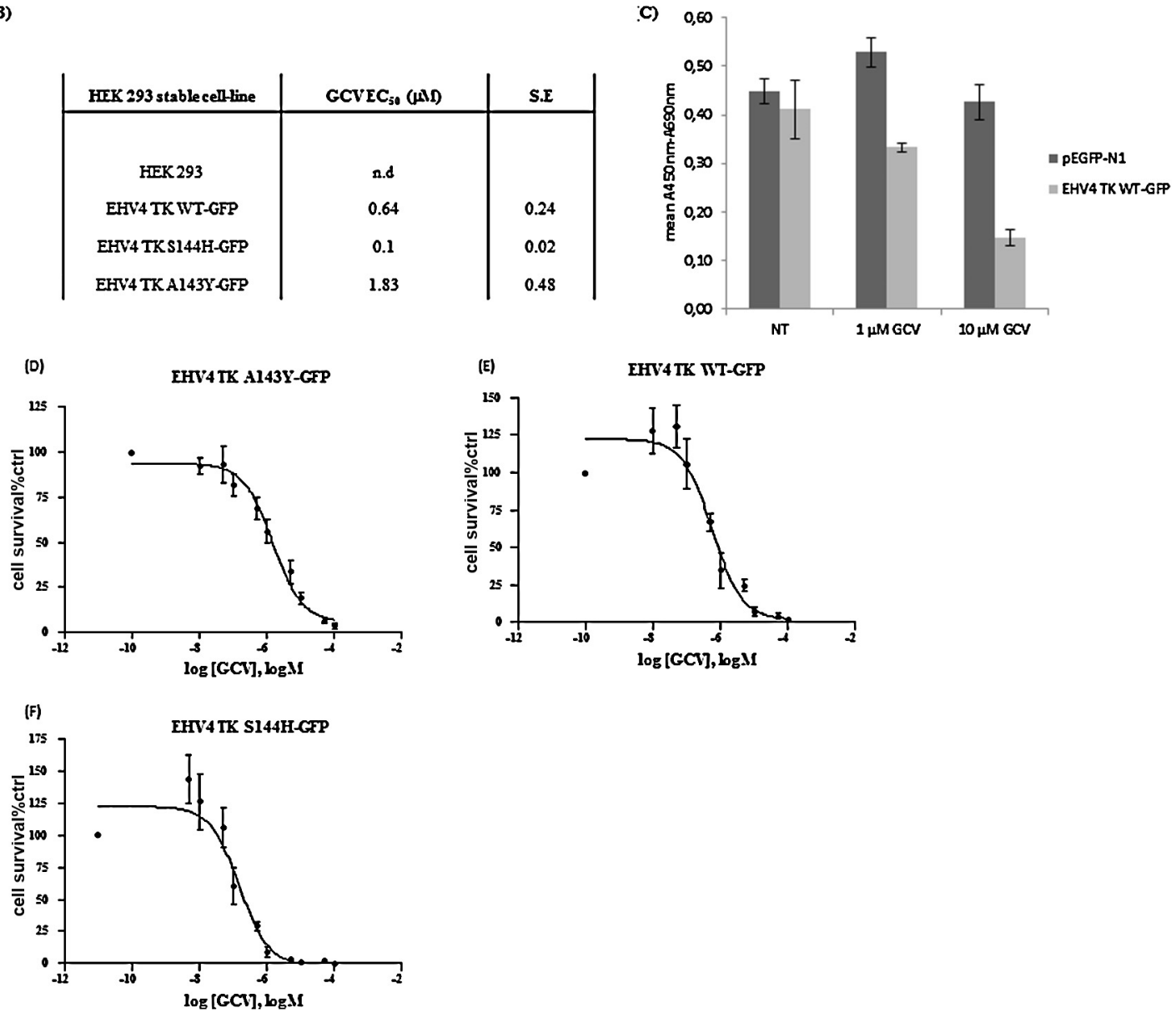

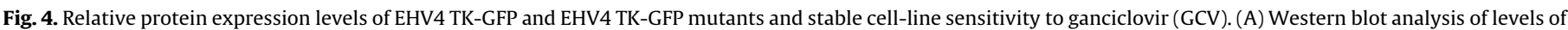

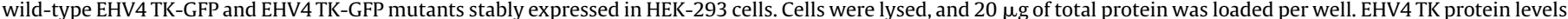

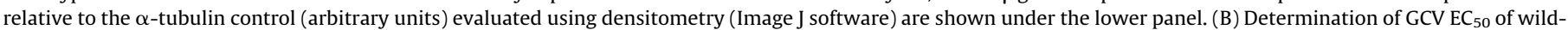

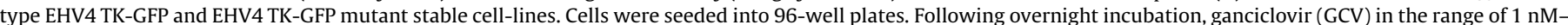

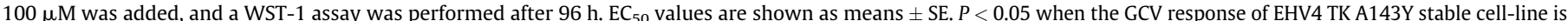

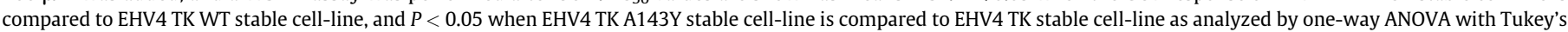

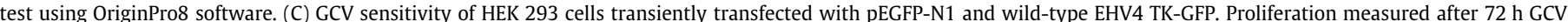

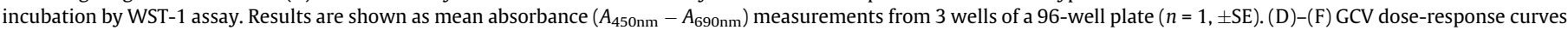

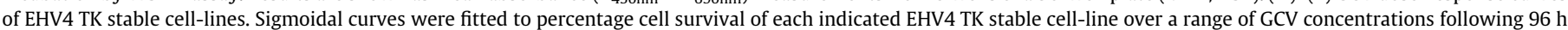
treatment using GraphPad Prism 5 software (nonlinear regression fitting; standard slope; three parameters; Hill-slope = 1.0, $n=3-7$, \pm SE).

studies of HSV1 TK have focused on improving the catalytic activity of HSV1 TK for ACV and GCV as substrates [18-22]. Our study aimed to modify the catalytic activity of another member or the herpes thymidine kinase family, the equine herpes virus-4 thymidine kinase (EHV4 TK). Previous research found that a human osteosarcoma cell-line expressing EHV4 TK was rendered 12-fold more sensitive to GCV compared to the same cell-line expressing HSV1 TK [23]. Therefore, in line with the studies focusing on HSV1 TK protein engineering, we investigated if it is possible to further improve EHV4 TK/GCV cytotoxicity by designed enzyme variants.
Firstly, we performed structure-based site-directed mutagenesis to enable EHV4 TK selectively to accept GCV as a substrate over the natural nucleoside dT. Residues A167 and A168 appeared to be important in the discrimination of GCV and dT binding to HSV1 TK $[18,22]$. Following sequence and structural comparisons of HSV1 TK and EHV4 TK [24], we proposed that the homologous mutations A143Y and $\mathrm{S} 144 \mathrm{H}$ would confer a shift from pyrimidine activity to predominantly purine activity. This was indeed the case: The EHV4 TK A143Y variant lost the ability to bind dT, and although the EHV4TK $\mathrm{S} 144 \mathrm{H}$ mutant still had residual dT activity, its $K_{\mathrm{m}}$ increased 


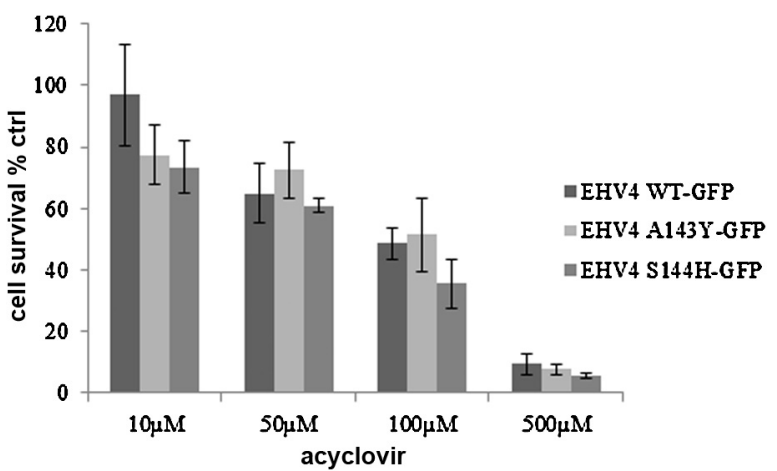

Fig. 5. Survival of EHV4 TK stable cell-lines following acyclovir (ACV) treatment. Cells were treated for $72 \mathrm{~h}$ with the indicated concentrations of ACV. Cytotoxicity was measured by the WST-assay. Columns represent the percentage change in cellsurvival relative to untreated control cells (ctrl) $(n=3, \pm \mathrm{SE})$.

more than 100 -fold. The high phosphorylation rate of GCV by wild-type EHV4 TK was maintained following the A143Y mutation, and was even increased 3-fold with the $\mathrm{S} 144 \mathrm{H}$ mutation. Although both mutations resulted in increased $K_{\mathrm{m}}$ values for GCV, it can be assumed that in a cellular context, the substrate competition between dT and GCV would be ruled out, resulting in an increase in the GCV phosphorylating activity, and thus increase the GCV-MP pool available to participate in the next step of the phosphorylation pathway. Importantly, the presence of the thymidine kinase-deficient enzyme variant does not perturb nucleotide levels, and will not affect cells in the absence of GCV.

We tested this hypothesis by generating mammalian cell-lines expressing EHV4 TK variants and wild-type EHV4 TK. For ease of handling, we chose the HEK 293 cell-line to generate stable cell-lines and a GFP fusion system for visualization. Initially, we encountered a curious hurdle, whereby our N-terminal GFP EHV4 TK wild-type construct when transiently transfected into HEK 293 cells resulted in the formation of 'polymeric structures'. As EHV4 TK does not possess a cellular localization signal sequence, we had expected to observe GFP-EHV4 TK uniformly distributed throughout the cytosol and nucleus. As this was not the case, we believe that, due to the N-terminal GFP fusion moiety, misfolding may occur as EHV4 TK is one of few proteins reported to form a slipknot during folding [27]. As the slipknot is proposed to be formed by the passing of the EHV4 TK N-terminal region into a structural opening, we constructed a C-terminal GFP fusion of EHV4 TK with the assumption that misfolding would in this case not occur. Indeed, upon transfection of HEK 293 cells with EHV4 TK WT-GFP, EHV4 TK A143Y-GFP or EHV4 TK S144H-GFP, a uniform cytosolic and nuclear expression was observed. Furthermore, the stable cell-lines created with these constructs displayed similar morphology and proliferation rates (Fig. 3).

Although Western blot analysis of EHV4 TK WT-GFP, EHV4 TK A143Y-GFP, and EHV4 TK S144H-GFP protein expression showed different levels, we tested their sensitivity to GCV. The viability of the stable cell-lines after $96 \mathrm{~h}$ following exposure to differing concentrations of GCV was measured by WST-1 assays, and dose-response curves were generated. Surprisingly, considering the protein expression levels, the $\mathrm{EC}_{50}$ values (Fig. 4B) derived from the dose-response curves indicated that the EHV4 TK S144H-GFP stable cell-line was more sensitive to GCV than that of the more highly expressing EHV4 TK WT-GFP stable cell-line. The $\mathrm{EC}_{50}$ value of EHV4 TK S144H-GFP $(0.1 \pm 0.02 \mu \mathrm{M})$ is lower than that of EHV4 TK WT-GFP $(0.6 \pm 0.2 \mu \mathrm{M})$, despite the fact that EHV4 TK WT-GFP is more highly expressed. In the case of the EHV4 TK A143Y-GFP stable cell-line, which showed the lowest EHV4 TK expression level, the $\mathrm{EC}_{50}$ value was measured at $1.8 \pm 0.5 \mu \mathrm{M}$.

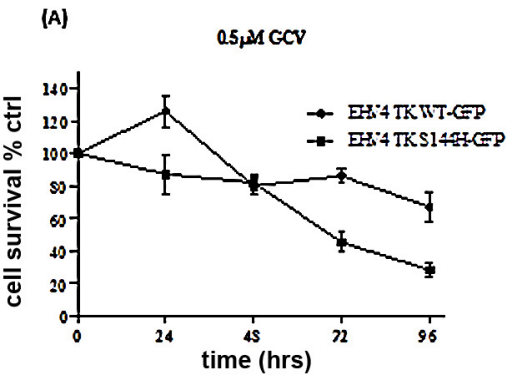

(c)

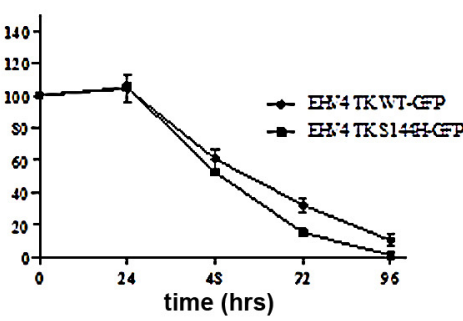

(日)

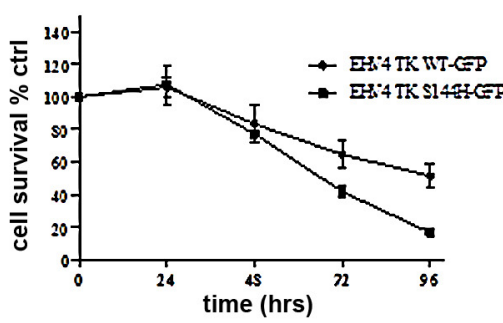

(D)
EHV4 TK S144H-GFP

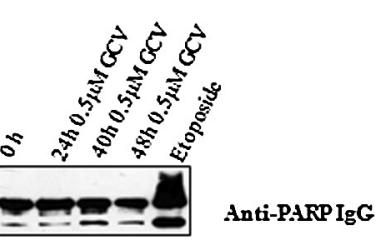

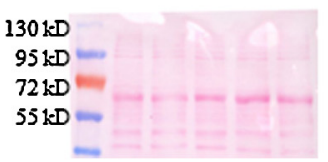

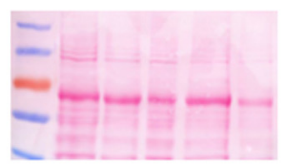

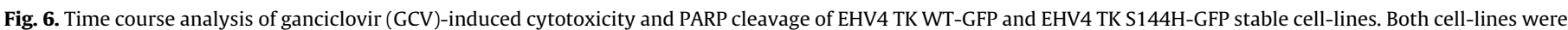

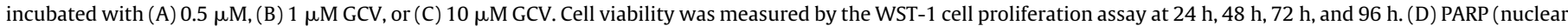

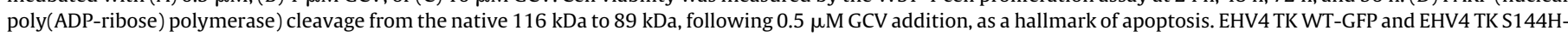

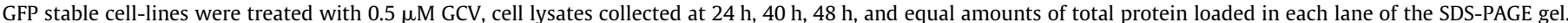

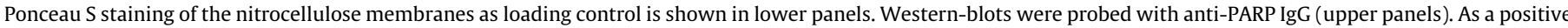
control to indicate apoptosis, HEK 293 cells were treated overnight with $25 \mu \mathrm{M}$ etoposide (topoisomerase inhibitor). 
Exposing each of the EHV4 TK stable cell-lines to the nucleoside analog prodrug ACV for $72 \mathrm{~h}$, resulted in significant cell death only at high concentrations $(500 \mu \mathrm{M})$ (Fig. 5). Previous studies have shown that not only does ACV have a different mechanism of cellular cytotoxicity compared to GCV, the ACV-TP metabolite has also very short intra-cellular half-life compared to GCV-TP [32,33]. Therefore, we expected that our EHV4 TK mutants would not confer increased ACV sensitivity compared to EHV4 TK WT. Taken together, these results indicate that HEK 293 cells expressing the EHV4 TK S144H-GFP mutant are more sensitive to GCV compared to HEK 293 cells expressing EHV4 TK WT-GFP or EHV4 TK A143YGFP, whereas sensitivity to ACV remains unchanged. It remains to be analyzed whether the higher $\mathrm{EC}_{50}$ value observed for $\mathrm{EHV} 4 \mathrm{TK}$ A143Y-GFP could be related its lower intracellular expression level.

Once GCV is phosphorylated by a viral thymidine kinase, in our case EHV4 TK, it is further phosphorylated by cellular kinases resulting in diphosphate (GCV-DP) and triphosphate (GCV-TP) forms [8]. Previously, it has been reported that GCV-TP is incorporated into cellular DNA, cells complete one round of mitosis, arrest in S-phase of the cell-cycle resulting in doublestranded breaks, and ultimately go into apoptosis [15,34]. The appearance of apoptotic cells at $24 \mathrm{~h}$, which increased greatly at 36-72 h has been reported following the incubation of a CHO cellline stably expressing HSV1-TK with GCV [15]. Our findings are in line with this apoptotic time-scale, as we observed in the case of both EHV4 TK WT-GFP and EHV4 TK S144H-GFP that at GCV concentrations in excess of the $\mathrm{EC}_{50}$ value, cell-death occurs from $24 \mathrm{~h}$ onwards (Fig. 6(A) and (C)). Furthermore, analysis of the cleavage of nuclear poly(ADP-ribose) polymerase (PARP) revealed that most cleavage occurred at the $40 \mathrm{~h}$ time-point (Fig. 6D).

Overall, our results indicate that the EHV4 TK S144H-GFP mutant, which preferentially phosphorylates the purine nucleoside analog GCV rather than dT, confers HEK 293 cells with increased sensitivity to GCV compared to EHV4 TK WT-GFP. Our thymidine kinase-deficient enzyme variant could be of particular interest for use in recently developed GCV-dependent suicide gene therapy systems that are based on controllable promoters that appear to be highly effective in certain tumors [35,36].

\section{Funding}

Supported by the Deutsche Forschungsgemeinschaft and the Max-Planck-Gesellschaft (T.M. and M.K.).

\section{Acknowledgements}

We thank Ursula Welscher-Altschäffel for excellent technical assistance and advice, and Christos S. Karamitros for help with molecular graphics. We extend our thanks to Professor Reinhard Lührmann (MPI for Biophysical Chemistry, Göttingen) for the use of the Zeiss LSM5 confocal microscope and the $\mu$ Quant microplate spectrophotometer (Bio-Tek Instruments).

\section{References}

[1] Moolten FL. Tumor chemosensitivity conferred by inserted herpes thymidine kinase genes: paradigm for a prospective cancer control strategy. Cancer Res 1986;46:5276-81.

[2] Culver KW, Van Gilder J, Link CJ, Carlstrom T, Buroker T, Yuh W, et al. Gene therapy for the treatment of malignant brain tumors with in vivo tumor transduction with the herpes simplex thymidine kinase gene/ganciclovir system. Hum Gene Ther 1994;5:343-79.

[3] Klatzmann D, Valery CA, Bensimon G, Marro B, Boyer O, Mokhtari K, et al. A phase I/II study of herpes simplex virus type 1 thymidine kinase "suicide" gene therapy for recurrent glioblastoma. Study Group on Gene Therapy for Glioblastoma. Hum Gene Ther 1998;9:2595-604.

[4] Rainov NG. A phase III clinical evaluation of herpes simplex virus type 1 thymidine kinase and ganciclovir gene therapy as an adjuvant to surgical resection and radiation in adults with previously untreated glioblastoma multiforme. Hum Gene Ther 2000;11:2389-401.

[5] Trask TW, Trask RP, Aguilar-Cordova E, Shine HD, Wyde PR, Goodman JC, et al. Phase I study of adenoviral delivery of the HSV-tk gene and ganciclovir administration in patients with current malignant brain tumors. Mol Ther 2000; 1:195-203.

[6] Cihova M, Altanerova V, Altaner C. Stem cell based cancer gene therapy. Mol Pharm 2011;8:1480-7.

[7] Choi KJ, Kim SU, Choi KC. Therapeutic potential of stem cells expressing suicide genes that selectively target human breast cancer cells: evidence that they exert tumoricidal effects via tumor tropism. Int J Oncol 2012;41:798-804 [review].

[8] Matthews T, Boehme R. Antiviral activity and mechanism of action of ganciclovir. Rev Infect Dis 1988;10(Suppl. 3):S490-4.

[9] St Clair MH, Lambe CU, Furman PA. Inhibition by ganciclovir of cell growth and DNA synthesis of cells biochemically transformed with herpesvirus genetic information. Antimicrob Agents Chemother 1987:31:844-9.

[10] Reardon JE. Herpes simplex virus type 1 and human DNA polymerase interactions with 2'-deoxyguanosine 5'-triphosphate analogues. Kinetics of incorporation into DNA and induction of inhibition. J Biol Chem 1989;264:19039-44.

[11] Halloran PJ, Fenton RG. Irreversible G2-M arrest and cytoskeletal reorganization induced by cytotoxic nucleoside analogues. Cancer Res 1998;58:3855-65.

[12] Wei SJ, Chao Y, Hung YM, Lin WC, Yang DM, Shih YL, et al. S- and G2-phase cell cycle arrests and apoptosis induced by ganciclovir in murine melanoma cells transduced with herpes simplex virus thymidine kinase. Exp Cell Res 1998;241:66-75.

[13] Rubsam LZ, Boucher PD, Murphy PJ, KuKuruga M, Shewach DS. Cytotoxicity and accumulation of ganciclovir triphosphate in bystander cells cocultured with herpes simplex virus type 1 thymidine kinase-expressing human glioblastoma cells. Cancer Res 1999;59:669-75.

[14] Rubsam LZ, Davidson BL, Shewach DS. Superior cytotoxicity with ganciclovir compared with acyclovir and 1-beta-D-arabinofuranosylthymine in herpes simplex virus-thymidine kinase-expressing cells: a novel paradigm for cell killing. Cancer Res 1998;58:3873-82.

[15] Tomicic MT, Thust R, Kaina B. Ganciclovir-induced apoptosis in HSV-1 thymidine kinase expressing cells: critical role of DNA breaks, Bcl-2 decline and caspase-9 activation. Oncogene 2002;21:2141-53.

[16] Wild K, Bohner T, Folkers G, Schulz GE. The structures of thymidine kinase from herpes simplex virus type 1 in complex with substrates and a substrate analogue. Protein Sci 1997;6:2097-106.

[17] Pilger BD, Perozzo R, Alber F, Wurth C, Folkers G, Scapozza L. Substrate diversity of herpes simplex virus thymidine kinase. Impact of the kinematics of the enzyme. J Biol Chem 1999;274:31967-73.

[18] Degreve B, Esnouf R, De Clercq E, Balzarini J. Selective abolishment of pyrimidine nucleoside kinase activity of herpes simplex virus type 1 thymidine kinase by mutation of alanine-167 to tyrosine. Mol Pharmacol 2000;58:1326-32.

[19] Black ME, Kokoris MS, Sabo P. Herpes simplex virus-1 thymidine kinase mutants created by semi-random sequence mutagenesis improve prodrugmediated tumor cell killing. Cancer Res 2001;61:3022-6.

[20] Degreve B, Esnouf R, De Clercq E, Balzarini J. Mutation of Gln125 to Asn selectively abolishes the thymidylate kinase activity of herpes simplex virus type 1 thymidine kinase. Mol Pharmacol 2001;59:285-93.

[21] Mercer KE, Ahn CE, Coke A, Compadre CM, Drake RR. Mutation of herpesvirus thymidine kinase to generate ganciclovir-specific kinases for use in cancer gene therapies. Protein Eng 2002;15:903-11.

[22] Balzarini J, Liekens S, Solaroli N, El Omari K, Stammers DK, Karlsson A Engineering of a single conserved amino acid residue of herpes simplex virus type 1 thymidine kinase allows a predominant shift from pyrimidine to purine nucleoside phosphorylation. J Biol Chem 2006;281:19273-79.

[23] Loubiere L, Tiraby M, Cazaux C, Brisson E, Grisoni M, Zhao-Emonet J, et al. The equine herpes virus 4 thymidine kinase is a better suicide gene than the human herpes virus 1 thymidine kinase. Gene Ther 1999;6:1638-42.

[24] Gardberg A, Shuvalova L, Monnerjahn C, Konrad M, Lavie A. Structural basis for the dual thymidine and thymidylate kinase activity of herpes thymidine kinases. Structure 2003;11:1265-77.

[25] Ho SN, Hunt HD, Horton RM, Pullen JK, Pease LR. Site-directed mutagenesis by overlap extension using the polymerase chain reaction. Gene 1989;77:51-9.

[26] Agarwal KC, Miech RP, Parks Jr RE. Guanylate kinases from human erythrocytes, hog brain, and rat liver. Methods Enzymol 1978;51:483-90.

[27] King NP, Yeates EO, Yeates TO. Identification of rare slipknots in proteins and their implications for stability and folding. J Mol Biol 2007;373:153-66.

[28] Immonen A, Vapalahti M, Tyynela K, Hurskainen H, Sandmair A, Vanninen R, et al. AdvHSV-tk gene therapy with intravenous ganciclovir improves survival in human malignant glioma: a randomised, controlled study. Mol Ther 2004; 10:967-72.

[29] Wang Y, Canine BF, Hatefi A. HSV-TK/GCV cancer suicide gene therapy by a designed recombinant multifunctional vector. Nanomedicine 2011;7:193-200.

[30] Matuskova M, Hlubinova K, Pastorakova A, Hunakova L, Altanerova V, Altaner C, et al. HSV-tk expressing mesenchymal stem cells exert bystander effect on human glioblastoma cells. Cancer Lett 2010;290:58-67.

[31] Yin X, Yu B, Tang Z, He B, Ren J, Xiao X, et al. Bifidobacterium infantis-mediated HSV-TK/GCV suicide gene therapy induces both extrinsic and intrinsic apoptosis in a rat model of bladder cancer. Cancer Gene Ther 2012;20(2):77-81.

[32] Thust R, Tomicic M, Klocking R, Wutzler P, Kaina B. Cytogenetic genotoxicity of anti-herpes purine nucleoside analogues in CHO cells expressing the thymidine kinase gene of herpes simplex virus type 1: comparison of ganciclovir, penciclovir and aciclovir. Mutagenesis 2000;15:177-84. 
[33] Vere Hodge RA, Perkins RM. Mode of action of 9-(4-hydroxymethylbut-1yl)guanine (BRL 39123) against herpes simplex virus in MRC-5 cells. Antimicrob Agents Chemother 1989;33:223-9.

[34] Ladd B, O'Konek JJ, Ostruszka LJ, Shewach DS. Unrepairable DNA double-strand breaks initiate cytotoxicity with HSV-TK/ganciclovir. Cancer Gene Ther 2011;18:751-9.
[35] Azatian A, Yu H, Dai W, Schneiders FI, Botelho NK, Lord RV. Effectiveness of HSV-tk suicide gene therapy driven by the Grp78 stress-inducible promoter in esophagogastric junction and gastric adenocarcinomas. J Gastrointest Surg 2009;13:1044-51.

[36] Isomoto H, Ohtsuru A, Braiden V, Iwamatsu M, Miki F, Kawashita Y, et al. Heatdirected suicide gene therapy mediated by heat shock protein promoter for gastric cancer. Oncol Rep 2006;15:629-35. 